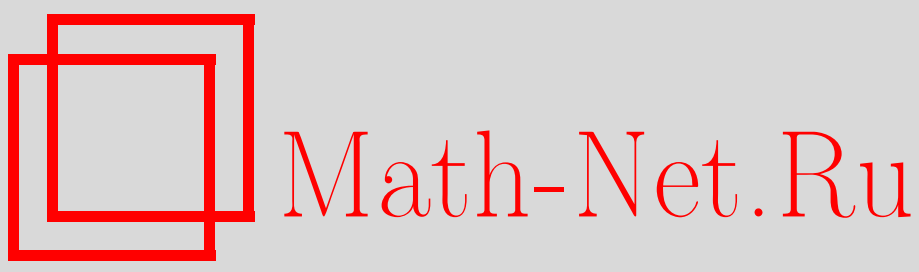

М. Вуоринен, О. Мартио, В. И. Рязанов, О локальном поведении пространственных квазирегулярных отображений, Изв. РАН. Сер. матем., 1998, том 62, выпуск $6,143-158$

DOI: https://doi.org/10.4213/im225

Использование Общероссийского математического портала Math-Net.Ru подразумевает, что вы прочитали и согласны с пользовательским соглашением

http://www . mathnet.ru/rus/agreement

Параметры загрузки:

IP : 52.205 .19 .152

26 апреля 2023 г., 15:56:45 
УДК 517.54

\author{
М. Вуоринен, О. Мартио, В.И. Рязанов
}

\title{
О локальном поведении пространственных квазирегулярных отображений
}

\begin{abstract}
Мы показываем, что локальная инъективность отображений имеет место, если их дилатационный тензор или матричная дилатация принадлежит классу $V M O$, исчезающей средней осцилляции, или классу $B M O$, ограниченной средней осцилляции, с достаточно малой $B M O$-нормой, или, наконец, хотя бы близки классу $V M O$ по $B M O$-норме.

Библиография: 29 наименований.
\end{abstract}

\section{§1. Введение}

$Q$-квазирегулярное отображение $f$ с ветвлением в пространстве не может иметь гладкий дилатационный тензор или $Q$, достаточно близкое к единице. Последнее свойство является результатом об устойчивости: для каждого $n \geqslant 3$ найдется $Q_{0}>1$ такое, что все непостоянные $Q$-квазирегулярные отображения $f: D \rightarrow \mathbb{R}^{n}$, $Q \leqslant Q_{0}$, являются локальными гомеоморфизмами (см., например, [1]-[3]). Локальная инъективность $f$ также имеет место, если дилатационный тензор $G_{f}$ является непрерывно дифференцируемым [4]. Этот результат был последовательно улучшен: в [5] для непрерывного $G_{f}$ и в [6] для апшроксимативно непрерьвного $G_{f}$.

В данной статье мы показываем, что локальная инъективность квазирегулярного отображения $f$ в пространстве тесно связана с $B M O$-поведением его дилатационного тензора $G_{f}$. Наш первый результат говорит, что если $G_{f}$ принадлежит классу $V M O$ исчезающей средней осциллящии, то $f$ локально инъективно. Далее мы показываем, что для каждого $n \geqslant 3$ и $Q>1$ найдется $\delta>0$ такое, что если $f: D \rightarrow \mathbb{R}^{n}$ является непостоянным $Q$-квазирегулярным отображением с $\left\|G_{f}\right\|_{*}<\delta$, то $f$ является локально инъективным. Здесь обозначение $\|\cdot\|_{*}$ относится к $B M O$-норме в $D$. Мы также показываем, что то же самое заключение получается, если $G_{f}$ близок к тензору $G$ класса $V M O$ в $B M O$-норме. Этот результат может быть использован, в частности, если $G_{f}$ равномерно близок к непрерывному тензору $G$.

Все эти результаты могут быть также сформулированы в терминах матричной дилатации $M_{f}$.

\section{§2. Определения и предварительные замечания}

Пусть $D \subset \mathbb{R}^{n}$ - область. Говорят, что вещественнозначная функция $\varphi \in L_{\mathrm{loc}}^{1}$ имеет ограниченную среднюю осщиллящию в $D$, если

$$
\|\varphi\|_{*}=\sup _{B \subset D} \frac{1}{|B|} \int_{B}\left|\varphi(x)-\varphi_{B}\right| d x<\infty
$$

(С) М. ВУоринен, О. МАртио, В. И. РЯЗАнов 1998 
где супремум берется над всеми шарами $B$ из $D$ и

$$
\varphi_{B}=\frac{1}{|B|} \int_{B} \varphi(x) d x
$$

обозначает среднее значение функции $\varphi$ над шаром $B \subset D$.

Ясно, что $L^{\infty} \subset B M O$ и $\|\varphi\|_{*} \leqslant 2\|\varphi\|_{\infty}$. Поскольку $\|c\|_{*}=0$ для каждой постоянной $c \in \mathbb{R}$, то мы можем отождествлять $\varphi \in B M O$ с $\varphi+$ const и таким образом рассматривать $B M O$ как подмножество $L_{\text {loc }}^{1} / \mathbb{R}$, состояшее из соответствующих классов эквивалентности функций из $L_{\mathrm{loc}}^{1} . B M O / \mathbb{R}$ с нормой $(2.1)$ является банаховым пространством. Пространство ВMO было введено Джоном и Ниренбергом [7] в 1961 г. и вскоре стало одним из центральных понятий гармонического анализа, комплексного анализа и теории дифференщиальных уравнений. В частности, о связях между $B M O$ и квазиконформными отображениями см. [8] и [9].

Далее мы говорим, что локально интегрируемая функция $\varphi: D \rightarrow \mathbb{R}$ имеет $u c$ чезаюшую среднюю осциллячию в точке $x_{0} \in D$, если

$$
\lim _{\varepsilon \rightarrow 0} \frac{1}{\Omega_{n} \varepsilon^{n}} \int_{\left|x-x_{0}\right|<\varepsilon}\left|\varphi(x)-\varphi_{\varepsilon}\left(x_{0}\right)\right| d x=0
$$

где $\Omega_{n}$ - объем единичного шара $\mathbb{B}^{n}$ в $\mathbb{R}^{n}$ и

$$
\varphi_{\varepsilon}\left(x_{0}\right)=\frac{1}{\Omega_{n} \varepsilon^{n}} \int_{\left|x-x_{0}\right|<\varepsilon} \varphi(x) d x
$$

- среднее значение функции $\varphi$ в шаре $B\left(x_{0}, \varepsilon\right)$.

Мы также говорим, что функция $\varphi: D \rightarrow \mathbb{R}$ имеет исчезаюшую среднюю осииллячию в $D$, и пишем $\varphi \in V M O$, если функция имеет это свойство в каждой точке $x_{0} \in D$.

В литературе (см., например, [10, с. 250]) часто используется следующее более ограничительное определение: $\varphi \in V M O$ означает, что, во-первых, $\varphi \in B M O$ и, во-вторых, предел в (2.3) является равномерным относительно параметра $x_{0} \in D$.

Заметим, что если $\varphi: D \rightarrow \mathbb{R}$ принадлежит $L^{\infty}$, то хорошо известное свойство аппроксимативной непрерывности функции $\varphi$ в точке $x_{0}$ (см. [11]) эквивалентно тому, что $x_{0}$ является точкой Лебега функции $\varphi$, т.е.

$$
\lim _{\varepsilon \rightarrow 0} \frac{1}{\Omega_{n} \varepsilon^{n}} \int_{\left|x-x_{0}\right|<\varepsilon}\left|\varphi(x)-\varphi\left(x_{0}\right)\right| d x=0,
$$

а это влечет то, что $\varphi$ имеет исчезающую среднюю осцилляцию в точке $x_{0}$.

Для матричнозначных функций $M(x), x \in D$, все перечисленные вьше понятия мы используем в поэлементном смысле. $\mathrm{K}$ примеру, $M \in B M O$, если

$$
\|M\|_{*}=\sup _{B \subset D} \frac{1}{|B|} \int_{B}\left\|M(x)-M_{B}\right\|_{1} d x<\infty
$$


где $M_{B}$ определено аналогично (2.2). Здесь и позже мы используем две нормы в пространстве $(n \times n)$-матриц $A=\left\{a_{i j}\right\}, a_{i j} \in \mathbb{R}$,

$$
\|A\|_{1}=\left(\sum_{i, j=1}^{n} a_{i j}^{2}\right)^{1 / 2}
$$

и

$$
\|A\|_{2}=\max _{h \in \mathbb{R}^{n} \backslash\{0\}} \frac{|A h|}{|h|} .
$$

Эти нормы удовлетворяют неравенствам

$$
\|A\|_{2} \leqslant\|A\|_{1} \leqslant n^{1 / 2}\|A\|_{2}
$$

Поэтому в (2.6) так же, как и в других $B M O$ - и $V M O$-условиях, может быть использована любая из этих норм.

В дальнейшем $\mathscr{O}^{+}(n)$ и $\mathscr{O}(n)$ обозначают группы $(n \times n)$-ортогональных матриц $U$, определяемых условием

$$
U^{*} U=I=U U^{*}
$$

с детерминантом, равным 1 и \pm 1 соответственно, где $I$ обозначает единичную матрицу, а $U^{*}$ - транспонированную для $U$.

Вообще, матрица $M$ называется унимодулярной, если $\operatorname{det} M=1$. Специальная линейная группа $\mathrm{SL}(n)$ есть мультипликативная группа всех унимодулярных $(n \times n)$-матриц над $\mathbb{R}$. Совокупность всех симметричных, положительно определенных матрищ из $\mathrm{SL}(n)$ обозначается через $S(n)$. Отметим, что $S(n), n \geqslant 2$, не является группой, поскольку произведение двух симметричных матрищ может быть несимметричной матрищей (см. $[12$, с. 24]). Симметризация матрищы $M \in \mathrm{SL}(n)$ определяется как

$$
G=M^{*} M \in S(n) .
$$

ЗАмечание 2.1 . Пусть $M_{1}, M_{2} \in \mathrm{SL}(n), n \geqslant 2$, и пусть $G_{1}$ и $G_{2}$ - симметризации $M_{1}$ и $M_{2}$ соответственно. Тогда равенство $M_{2}=U M_{1}, U \in \mathscr{O}^{+}(n)$, является необходимым и достаточным условием для того, чтобы $G_{1}=G_{2}$.

В дальнейшем $\mathrm{SL}_{q}(n)$ и $S_{q}(n)$ обозначают подмножества $\mathrm{SL}(n)$ и $S(n)$ всех матрищ $A$ с $\|A\|_{2} \leqslant q$.

Непрерывное отображение $f: D \rightarrow \mathbb{R}^{n}$ класса $W_{n, \text { loc }}^{1}$ с якобианом $J_{f}(x)>0$ почти всюду (п.в.) назьвается $Q$-квазирегулярным, $Q \geqslant 1$, если его локальная дилатация

$$
K_{f}(x)=\left\|f^{\prime}(x)\right\|_{2}^{n} / J_{f}(x) \leqslant Q \text { п.в. }
$$

Здесь через $f^{\prime}(x)$ и $J_{f}(x)$ мы обозначаем, как обычно, якобиеву матрицу отображения $f$ и ее детерминант $J_{f}(x)=\operatorname{det} f^{\prime}(x)$ соответственно. 
Нормированная якобиева матрица непостоянного квазирегулярного отображения $f$ определяется как

$$
M_{f}(x)=f^{\prime}(x) / J_{f}(x)^{1 / n} \text { п.в., }
$$

а симметризованная нормированная якобиева матрица - как

$$
G_{f}(x)=M_{f}^{*}(x) M_{f}(x),
$$

где, как и выше, $M^{*}$ - транспонированная матрица для $M$. Отметим, что (2.13) сначала задается в каждой регулярной точке $x \in D$, т.е. в точках, где $f$ дифференцируемо и $J_{f}(x) \neq 0$. Затем мы полагаем $M_{f}(x)=I$ в остальных точках $D$, чтобы завершить определение. Мы также называем $M_{f}(x)$ и $G_{f}(x)$ соответственно матричной дилатацией и дилатачионным тензором отображения $f$ в точке $x$ (см. [13], [14]).

Как легко видеть, $M_{f}(x) \in \mathrm{SL}_{q}(n)$ и $G_{f}(x) \in S_{q^{2}}(n)$, где $q=Q^{1 / n}$ для $Q$-квазирегулярных отображений. В частности, отсюда мы получаем следуюшие верхние границы для всех элементов матричной дилатации $\left|m_{f}^{i, k}(x)\right| \leqslant Q^{1 / n}$ и дилатационного тензора $\left|g_{f}^{i, k}(x)\right| \leqslant Q^{2 / n}, i, k=1,2, \ldots, n$.

Впоследствии мы используем пространственный аналог теоремы сходимости Берса-Боярского (ср., например, [15]-[17]), который был установлен в работе [18] .

Tеорема 2.1. Пусть $f$ u $f_{j}: D \rightarrow \mathbb{R}^{n}, n \geqslant 3, j=1,2, \ldots,-$ непостоянные $Q$-квазирегулярные отображения, и пусть $G_{j}$ - их дилатационные тензоры. Предположим, что $f_{j} \rightarrow f$ локально равномерно $и G_{j}(x) \rightarrow G_{0}(x)$ почти всюду при $j \rightarrow \infty$. Тогда

$$
G(x)=G_{0}(x) \quad \text { n. } 8 .
$$

Отсюда и из замечания 2.1 мы немедленно получаем также еще один вариант теоремы.

СлеДСТВИе 2.1. Пусть $f$ u $f_{j}: D \rightarrow \mathbb{R}^{n}, n \geqslant 3, j=1,2, \ldots,-$ непостоянные квазирегулярные отображения, и пусть $M$ и $M_{j}$ - их матричные дилатачии. Предположим, что $f_{j} \rightarrow f$ локально равномерно и $U_{j}(x) \cdot M_{j}(x) \rightarrow M_{0}(x)$ почти всюду при $j \rightarrow \infty$. Тогда

$$
M(x)=U(x) \cdot M_{0}(x) \quad \text { n.e. }
$$

Здесь $U(x)$ и $U_{j}(x)$ - некоторые ортогональнье матриць.

Другим способом следствие 2.1 было доказано в работе [19]. Отметим, что теорема 2.1 также может быть получена из следствия 2.1, но не совсем тривиальным способом.

Нам также нужна лемма из [6]. Пусть $f: D \rightarrow \mathbb{R}^{n}$-непостоянное $Q$-квазирегулярное отображение, и пусть $x_{0} \in D$. Для $0<\rho<\operatorname{dist}\left(x_{0}, \partial D\right)$ мы полагаем

$$
l\left(x_{0}, f, \rho\right)=\inf _{\left|x-x_{0}\right|=\rho}\left|f(x)-f\left(x_{0}\right)\right|
$$


И

$$
L\left(x_{0}, f, \rho\right)=\sup _{\left|x-x_{0}\right|=\rho}\left|f(x)-f\left(x_{0}\right)\right| .
$$

Напомним (см. [20]-[22]), что

$$
\limsup _{\rho \rightarrow 0} \frac{L\left(x_{0}, f, \rho\right)}{l\left(x_{0}, f, \rho\right)} \leqslant C<\infty .
$$

Здесь $C$ зависит только от $n$ и произведения $i_{f}\left(x_{0}\right) Q$, где $i_{f}\left(x_{0}\right)$ обозначает локальньй топологический индекс отображения $f$ в точке $x_{0}$. Другими словами, из (2.18) ясно, что искажение инфинитезимальных сфер ограничено постоянной $C$.

Нам нужно аналогичное утверждение об искажении инфинитезимальных сферических колец.

Лемма 2.1. Пусть $f: D \rightarrow \mathbb{R}^{n}, \quad n \geqslant 2$, - непостоянное $Q$-квазирегулярное отображсеие. Тогда имеют место неравенства

$$
\begin{aligned}
C^{-2} \Lambda^{\alpha} & \leqslant \liminf _{\rho \rightarrow 0} \frac{l\left(x_{0}, f, \rho \Lambda\right)}{L\left(x_{0}, f, \rho\right)} \leqslant \Lambda^{\beta}, \\
\Lambda^{\alpha} & \leqslant \limsup _{\rho \rightarrow 0} \frac{L\left(x_{0}, f, \rho \Lambda\right)}{l\left(x_{0}, f, \rho\right)} \leqslant C^{2} \Lambda^{\beta}
\end{aligned}
$$

для всех $\Lambda \geqslant 1$ слева и для всех $\Lambda>\gamma$ справа, где

$$
\alpha=Q^{-1}, \quad \beta=\left(i_{f}\left(x_{0}\right) Q\right)^{\frac{1}{n-1}}, \quad \gamma=C^{2 Q}>1
$$

Здесь $C$ - та жее самая постоянная, что и в (2.18), зависящая только от $n$ $i_{f}\left(x_{0}\right) Q$.

Для замкнутости изложения мы завершаем эту секцию доказательством последнего утверждения.

ДоКАЗАТЕЛЬСТво ЛЕммы 2.1. Без потери общности мы можем предполагать, что $f(0)=x_{0}=0 \in D$.

Пусть $\rho>0$ и $E_{\rho, \Lambda}-$ конденсатор $(\overline{B(0, \rho)}, B(0, \rho \Lambda))$. Тогда согласно [24, c. 15,29$]$

$$
\operatorname{cap} f\left(E_{\rho, \Lambda}\right) \leqslant Q^{n-1} \operatorname{cap} E_{\rho, \Lambda} .
$$

Кроме того, с одной стороны,

$$
\operatorname{cap} E_{\rho, \Lambda}=\frac{\omega_{n-1}}{(\log \Lambda)^{n-1}}
$$

с другой стороны, в силу свойства монотонности емкости

$$
\operatorname{cap} f\left(E_{\rho, \Lambda}\right) \geqslant \frac{\omega_{n-1}}{\left(\log \frac{L(0, f, \rho \Lambda)}{l(0, f, \rho)}\right)^{n-1}},
$$


где $\omega_{n-1}-(n-1)$-мерная площадь единичной сферы в $\mathbb{R}^{n}$. Таким образом,

$$
\frac{L(0, f, \rho \Lambda)}{l(0, f, \rho)} \geqslant \Lambda^{\frac{1}{Q}}
$$

и левое неравенство в (2.20) доказано. Используя (2.18), мы получаем отсюда левое неравенство в (2.19).

Обозначим через $U_{t}$ связную компоненту прообраза $f^{-1}(B(0, t))$, содержащую начало. По лемме 2.9 работы $[20$, с. 9,10$]$ ее диаметр $d\left(U_{t}\right) \rightarrow 0$ при $t \rightarrow 0$ и сушествует $\delta>0$ такое, что $U_{t}$ является нормальной областью, а $\partial U_{t}=U_{\delta} \cap$ $f^{-1}(\partial B(0, t))$ для $t \in(0, \delta], U_{t_{1}} \subseteq U_{t_{2}}$ и $U_{t_{2}} \backslash \overline{U_{t_{1}}}$ является кольцевой областью для всех $0<t_{1}<t_{2} \leqslant \delta$.

Теперь для $\Lambda>\gamma=C^{2 Q}$ левая часть неравенства (2.19) больше единищы. Поэтому для малых $\rho$ конденсаторы

$$
\mathscr{E}_{\rho, \Lambda}=\left(\overline{U_{L(0, f, \rho)}}, U_{l(0, f, \rho \Lambda)}\right)
$$

и

$$
f\left(\mathscr{E}_{\rho, \Lambda}\right)=(\overline{B(0, L(0, f, \rho))}, B(0, l(0, f, \rho \Lambda)))
$$

корректно определены. Кроме того,

$$
\operatorname{cap} f\left(\mathscr{E}_{\rho, \Lambda}\right)=\frac{\omega_{n-1}}{\left(\log \frac{l(0, f, \rho \Lambda)}{L(0, f, \rho)}\right)^{n-1}}
$$

и монотонность емкостей влечет неравенство

$$
\operatorname{cap} \mathscr{E}_{\rho, \Lambda} \geqslant \frac{\omega_{n-1}}{(\log \Lambda)^{n-1}}
$$

По теореме 6.2 из [20]

$$
\operatorname{cap} \mathscr{E}_{\rho, \Lambda} \leqslant i_{f}(0) Q \operatorname{cap} f\left(\mathscr{E}_{\rho, \Lambda}\right)
$$

Сравнивая (2.22)-(2.24), мы видим, что

$$
\frac{l(0, f, \rho \Lambda)}{L(0, f, \rho)} \leqslant \Lambda^{\beta},
$$

т.е. правое неравенство в (2.19) доказано.

Наконец, используя снова неравенство (2.18), мы приходим к правому неравенству в (2.20). 


\section{§ 3. $V M O$ и инъективность}

В этом параграфе мы показываем, что если дилатационньй тензор пространственного отображения принадлежит классу $V M O$, то $f$ не имеет ветвления.

Теорема 3.1. Пусть $f: D \rightarrow \mathbb{R}^{n}, n \geqslant 3,-$ непостоянное квазирегулярное отображение, и пусть дилатационный тензор $G_{f}(x)$ или матричная дилатачия $M_{f}(x)$ принадлежит классу VMO. Тогда отображсение $f$ является локальным гомеоморфизмом области $D$.

Доказательство теоремы редуцируется к следующей лемме.

Лемма 3.1. Пусть $f: D \rightarrow \mathbb{R}^{n}, \quad n \geqslant 3$, - непостоянное квазирегулярное отображсение и дилатачионный тензор $G_{f}(x)$ или матричная дилатаиия $M_{f}(x)$ имеют исчезающую среднюю осииллячию в точке $x_{0} \in D$. Тогда отображение $f$ является гомеоморфизмом в некоторой окрестности точки $x_{0}$.

ЗАмечАнИЕ 3.1. Эти результаты имеют различные следствия:

a) $f$ является гомеоморфизмом в окрестности точки $x_{0}$, если $G_{f}$ непрерьвен или аппроксимативно непрерывен в точке $x_{0}$;

b) $f$ является локальным гомеоморфизмом области $D$, если $G_{f}$ непрерьвен или аппроксимативно непрерывен в $D$ (см. [5] и [6] соответственно);

с) более того, ввиду известной теоремы Зорича [23] $f: \mathbb{R}^{n} \rightarrow \mathbb{R}^{n}, n \geqslant 3,-$ гомеоморфизм $\mathbb{R}^{n}$, если $G_{f}$ - класса $V M O$;

d) в частности, $f: \mathbb{R}^{n} \rightarrow \mathbb{R}^{n}, n \geqslant 3$, является гомеоморфизмом $\mathbb{R}^{n}$, если $G_{f}$ непрерывен или аппроксимативно непрерывен в $\mathbb{R}^{n}$.

Аналогичные результаты имеют место в терминах матричной дилатации $M_{f}$.

ДОКАЗАТЕЛЬСТво ЛЕММЫ 3.1. ПреДположим, что

$$
i_{f}\left(x_{0}\right)>1
$$

где $i_{f}\left(x_{0}\right)$ обозначает, как обычно, локальный топологический индекс отображения $f$ в точке $x_{0}$, и пусть, для примера, $G_{f}(x)$ имеет исчезаюшую среднюю осцилляцию в $x_{0}$. Тогда мы имеем

$$
\lim _{\varepsilon \rightarrow 0} \frac{1}{\Omega_{n} \varepsilon^{n}} \int_{\left|x-x_{0}\right|<\varepsilon}\left\|G_{f}(x)-M_{\varepsilon}\right\|_{1} d x=0 ; \quad M_{\varepsilon}=\frac{1}{\Omega_{n} \varepsilon^{n}} \int_{\left|x-x_{0}\right|<\varepsilon} G_{f}(x) d x .
$$

Для $\varepsilon>0$ рассмотрим семейство $Q$-квазирегулярных отображений $h_{\varepsilon}$ : $\mathbb{B}^{n} \rightarrow \mathbb{B}^{n}$,

$$
h_{\varepsilon}(z)=\frac{f\left(x_{0}+\varepsilon z\right)-f\left(x_{0}\right)}{L\left(x_{0}, f, \varepsilon\right)},
$$

где $L$ определено (2.17). Отображение $h_{\varepsilon}$ имеет дилатационный тензор

$$
g_{\varepsilon}(z)=G_{f}\left(x_{0}+\varepsilon z\right)
$$


и поэтому мы получаем

$$
\lim _{\varepsilon \rightarrow 0} \int_{\mathbb{B}^{n}}\left\|g_{\varepsilon}(z)-M_{\varepsilon}\right\|_{1} d z=0, \quad M_{\varepsilon}=\frac{1}{\Omega_{n}} \int_{\mathbb{B}^{n}} g_{\varepsilon}(z) d z .
$$

Все элементы матриц $g_{\varepsilon}(z)$ и, следовательно, $M_{\varepsilon}$ ограничены числом $Q^{2 / n}$. Таким образом, найдется последовательность $\varepsilon_{j} \rightarrow 0$ такая, что

$$
\lim _{j \rightarrow \infty} M_{\varepsilon_{j}}=G_{0}
$$

и $g_{\varepsilon_{j}}(z) \rightarrow G_{0}$ п.в., где $G_{0} \in S_{q}(n), q=Q^{2 / n}$.

Пусть $H_{j}=h_{\varepsilon_{j}}$. Так как $H_{j}\left(\mathbb{B}^{n}\right) \subset \mathbb{B}^{n}$, последовательность отображений $\left(H_{j}\right)$ является равностепенно непрерывной (см. [24, с. 10]). Поэтому по теореме Арцела-Асколи семейство $H_{j}$ является нормальным, и без потери общности мы можем также предполагать, что $H_{j} \rightarrow h$ при $j \rightarrow \infty$ локально равномерно в $\mathbb{B}^{n}$, где $h$ по известной теореме Решетняка (см., например, [3, с. 180]) является $Q$-квазирегулярным отображением с $h(0)=0$. Покажем, что $h \neq$ const.

Действительно,

$$
\left|h_{\varepsilon_{j}}(z)\right|=\frac{\left|f\left(x_{0}+\varepsilon_{j} z\right)-f\left(x_{0}\right)\right|}{L\left(x_{0}, f, \varepsilon_{j}\right)} \geqslant \frac{l\left(x_{0}, f, \varepsilon_{j}|z|\right)}{L\left(x_{0}, f, \varepsilon_{j}\right)},
$$

и, переходя к пределу при $j \rightarrow \infty$, мы получаем по (2.20) неравенство

$$
|h(z)| \geqslant C^{-2}|z|^{\beta}
$$

для $0<|z|<C^{-2 Q}<1$, где $\beta=\left(i_{f}\left(x_{0}\right) \cdot Q\right)^{\frac{1}{n-1}}$.

Таким образом, по теореме $2.1 h$ имеет постоянный дилатационный тензор $G_{0}$ в $\mathbb{B}^{n}$. Пусть $M$ - матрища, определенная соотношением $M^{*} M=G_{0}$ (см. замечание 2.1 ), и пусть $A: \mathbb{R}^{n} \rightarrow \mathbb{R}^{n}$ - соответствующее аффинное отображение, $A(x)=M \cdot x$. Тогда отображение $H=h \circ A^{-1}: \mathbb{B}^{n} \rightarrow \mathbb{R}^{n}$ является 1-квазирегулярным отображением в эллипсоиде $A\left(\mathbb{B}^{n}\right)$ и по известной теореме Лиувилля $H$-преобразование Мёбиуса (см., например, [3], [23]-[25]). Поэтому $i_{h}(0)=1$ и, таким образом, $i_{H_{j}}(0)=1$ для больших $j$ (см. [2, с. 24]). Это противоречит предположению (3.1). Тем самым лемма 3.1 доказана.

\section{§4. ВMO-норма и инъективность}

Здесь мы показываем, что пространственные $Q$-квазирегулярные отображения являются инъективными, если $B M O$-нормы дилатационного тензора или матричной дилатации достаточно малы.

Tеорема 4.1. Пусть $n \geqslant 3 u Q>1$. Тогда найдется $\delta>0$ maкое, ито каждое непостоянное $Q$-квазирегулярное отображсение $f: D \rightarrow \mathbb{R}^{n}, D \subset \mathbb{R}^{n}$, локально ингективно, если

$$
\left\|G_{f}(x)\right\|_{*, D}<\delta
$$

или аналогичное неравенство имеет место для $M_{f}$.

В частном случае $D=\mathbb{R}^{n}$ теорема Зорича влечет глобальную инъективность таких квазирегулярных отображений. 
СлЕДСТВИЕ 4.1. Пусть $n \geqslant 3 u Q>1$. Тогда найдется $\delta>0$ maкое, ито каждое непостоянное $Q$-квазирегулярное отображсение $f: \mathbb{R}^{n} \rightarrow \mathbb{R}^{n}$ является гомеоморфизмом, т.е. квазиконформным отображением $\mathbb{R}^{n}$, если

$$
\left\|G_{f}(x)\right\|_{*, \mathbb{R}^{n}}<\delta
$$

или имеет место аналогичное неравенство для $M_{f}$.

ДоКАЗАТЕЛЬСТво тЕОРЕмЫ 4.1. Допустим, что утверждение теоремы неверно, например, относительно дилатационного тензора. Тогда сушествует последовательность $Q$-квазирегулярных отображений $f_{j}: D_{j} \rightarrow \mathbb{R}^{n}$ с

$$
\left\|G_{f_{j}}(x)\right\|_{*, D_{j}}<1 / j
$$

и последовательность точек $x_{j} \in D_{j}$ с локальньми топологическими индексами

$$
2 \leqslant i_{f_{j}}\left(x_{j}\right) \leqslant 9 Q^{n-1}
$$

(см. [2, с. 23]). Другими словами, здесь мы использовали тот факт, что локальный топологический индекс $Q$-квазирегулярного отображения не может быть равномерно сколь угодно большим на $B_{f}$.

В частности, для всех $0<\varepsilon<\Delta_{j}=\operatorname{dist}\left(x_{j}, \partial D_{j}\right)$ мы получаем из (4.3), что

$$
\frac{1}{\Omega_{n} \varepsilon^{n}} \int_{\left|x-x_{j}\right|<\varepsilon}\left\|G_{f_{j}}(x)-M_{\varepsilon}\right\|_{1} d x<\frac{1}{j}, \quad M_{\varepsilon}=\frac{1}{\Omega_{n} \varepsilon^{n}} \int_{\left|x-x_{j}\right|<\varepsilon} G_{f_{j}}(x) d x .
$$

Рассмотрим семейство $Q$-квазирегулярных отображений $H_{j, \varepsilon}: \mathbb{B}^{n} \rightarrow \mathbb{B}^{n}$,

$$
H_{j, \varepsilon}(z)=\frac{f_{j}\left(x_{j}+\varepsilon z\right)-f_{j}\left(x_{j}\right)}{L\left(x_{j}, f_{j}, \varepsilon\right)},
$$

где $0<\varepsilon<\Delta_{j}$. Отметим, что

$$
\left|H_{j, \varepsilon}(z)\right| \geqslant \frac{l\left(x_{j}, f_{j}, \varepsilon|z|\right)}{L\left(x_{j}, f_{j}, \varepsilon\right)}
$$

и по лемме 2.1 для каждого фиксированного $j=1,2, \ldots$ и $z_{0} \in \mathbb{B}^{n}, 0<\left|z_{0}\right|<$ $C^{-2 Q}$, найдется $0<\varepsilon_{j}<\Delta_{j}$ такое, что $\left|h_{j}\left(z_{0}\right)\right| \geqslant C^{-2}\left|z_{0}\right|^{\beta}-1 / j$, где $\beta=$ $\left(9 Q^{n}\right)^{\frac{1}{n-1}}$ и $h_{j}(z)=H_{j, \varepsilon_{j}}(z)$.

Отображения $h_{j}$ имеют дилатационные тензоры $g_{j}(z)=G_{f_{j}}\left(x_{j}+\varepsilon_{j} z\right)$, и $(4.5)$ влечет

$$
\lim _{j \rightarrow \infty} \int_{\mathbb{B}^{n}}\left\|g_{j}(z)-m_{j}\right\|_{1} d z=0, \quad m_{j}=\frac{1}{\Omega_{n}} \int_{\mathbb{B}^{n}} g_{j}(z) d z .
$$

Отметим, что все элементы матрищ $g_{j}(z)$ и, следовательно, $m_{j}$ ограничены числом $Q^{2 / n}$. Поэтому без потери общности мы можем также предполагать, что 
$\lim _{j \rightarrow \infty} m_{j}=G_{0} \in S_{q}(n), q=Q^{2 / n}$, и $\lim _{j \rightarrow \infty} g_{j}(z)=G_{0}$ почти всюду в единичном шаре $\mathbb{B}^{n}$.

Так как $h_{j}\left(\mathbb{B}^{n}\right) \subset \mathbb{B}^{n}$, последовательность $h_{j}$ является равностепенно непрерывной. Следовательно, без потери обшности мы можем предполагать, что $h_{j} \rightarrow h$ локально равномерно в $\mathbb{B}^{n}$ при $j \rightarrow \infty$, где $h$ - некоторое $Q$-квазирегулярное отображение с $h(0)=0$. По построению $\left|h\left(z_{0}\right)\right| \geqslant C^{-2}\left|z_{0}\right|^{\beta}>0$ в зафиксированной вьше точке $z_{0} \in \mathbb{B}^{n} \backslash\{0\}$ и, следовательно, $h$ не является постоянной. Поэтому $h$, с точностью до преобразования Мёбиуса, есть аффинное отображение с постоянным дилатационным тензором $G_{0}$. Следовательно, $i_{h}(0)=1$ и, таким образом, $i_{h_{j}}(0)=1$ для больших $j$. Последнее противоречит предположению (4.3), и доказательство теоремы завершено для $G_{f}$.

Доказательство для $M_{f}$ аналогично.

\section{§5. Близость в $B M O$-норме и инъективность}

Наш метод позволяет продвинуться еще дальше. Если $G$ - матричнозначная функция в области $D \subset \mathbb{R}^{n}$ и если $S$ - некоторый класс таких функций, то мы пишем

$$
d_{*}(G, S)=\inf _{M \in S}\|G-M\|_{*} .
$$

Tеорема 5.1. Пусть $n \geqslant 3 u Q>1$. Тогда найдется $\delta>0$ maкoе, что каждое непостоянное $Q$-квазирегулярное отображение $f: D \rightarrow \mathbb{R}^{n}$ является локально инбективным, если $d_{*}\left(G_{f}, V M O\right)<\delta$ или $d_{*}\left(M_{f}, V M O\right)<\delta$.

ЗАмечаниЕ 5.1. Теорема 5.1 имеет множество интересных следствий.

a) Пусть $C$ - класс непрерывных матричнозначных функций в области $D$. Поскольку $C \subset V M O, f$ является локально инъективным в предположении, что $d_{*}\left(G_{f}, C\right)<\delta$.

б) Если $f: \mathbb{R}^{n} \rightarrow \mathbb{R}^{n}$, то соответствующие результаты о глобальной инъективности $f$ могут быть получены из теоремы Зорича.

в) Пусть $A P C$ - класс аппроксимативно непрерывных матричнозначных функций в $D$. Тогда $f$ локально инъективно, если $d_{\infty}\left(G_{f}, A P C\right)$ достаточно мало. В частности, это имеет место, если $d_{\infty}\left(G_{f}, C\right)$ достаточно мало (см. [6]).

Аналогичные результаты могут быть сформулированы в терминах матричной дилатации $M_{f}$.

ДоКАЗАТЕЛЬСТВО ТЕОРЕмЫ 5.1. Допустим, что утверждение теоремы неверно, например, относительно дилатационного тензора. Тогда найдутся последовательности $Q$-квазирегулярных отображений $f_{j}: D_{j} \rightarrow \mathbb{R}^{n}$, заданных в областях $D_{j} \subset \mathbb{R}^{n}$, и матричнозначных функций $M_{j} \in V M O$ такие, что

$$
\left\|G_{f_{j}}-M_{j}\right\|_{*, D_{j}}<1 / j
$$

и последовательность точек $x_{j} \in D_{j}$ с локальньми топологическими индексами

$$
2 \leqslant i_{f_{j}}\left(x_{j}\right) \leqslant 9 Q^{n-1}
$$


(см. [2, с. 23]).

Теперь следуюшие шаги доказательства являются аналогичными шагам предыдущих доказательств. Рассмотрим семейство $Q$-квазирегулярных отображений $r_{j}: \mathbb{B}^{n} \rightarrow \mathbb{B}^{n}$,

$$
H_{j, \varepsilon}(z)=\frac{f_{j}\left(x_{j}+\varepsilon z\right)-f_{j}\left(x_{j}\right)}{L\left(x_{j}, f_{j}, \varepsilon\right)},
$$

где $0<\varepsilon<\Delta_{j}=\operatorname{dist}\left(x_{j}, \partial D_{j}\right)$.

Отметим, что

$$
\left|H_{j, \varepsilon}(z)\right| \geqslant \frac{l\left(x_{j}, f_{j}, \varepsilon|z|\right)}{L\left(x_{j}, f_{j}, \varepsilon\right)},
$$

и по лемме 2.1 для каждого фиксированного $j=1,2, \ldots$ и $z_{0} \in \mathbb{B}^{n}, 0<\left|z_{0}\right|<$ $C^{-2 Q}$, найдется $0<\delta_{j}<\Delta_{j}$ такое, что $\left|H_{j, \varepsilon}\left(z_{0}\right)\right| \geqslant C^{-2}\left|z_{0}\right|^{\beta}-1 / j$ для всех $0<\varepsilon<\delta_{j}$, где $\beta=\left(9 Q^{n}\right)^{\frac{1}{n-1}}$.

Выбирая достаточно малое $0<\varepsilon_{j}<\delta_{j}$ такое, что

$$
\frac{1}{\Omega_{n} \varepsilon_{j}^{n}} \int_{\left|x-x_{j}\right|<\varepsilon_{j}}\left\|M_{j}(x)-m_{j}\right\|_{1} d x<\frac{1}{j}, \quad m_{j}=\frac{1}{\Omega_{n} \varepsilon_{j}^{n}} \int_{\left|x-x_{j}\right|<\varepsilon_{j}} M_{j}(x) d x,
$$

мы получаем из (5.1), что

$$
\lim _{j \rightarrow \infty} \frac{1}{\Omega_{n} \varepsilon_{j}^{n}} \int_{\left|x-x_{j}\right|<\varepsilon_{j}}\left\|G_{f_{j}}(x)-m_{j}\right\|_{1} d x=0 .
$$

Пусть $h_{j}(z)=H_{j, \varepsilon_{j}}(z)$. Дилатационные тензоры $g_{j}(z)=G_{f_{j}}\left(x_{j}+\varepsilon_{j} z\right)$ отображений $h_{j}$ удовлетворяют по (5.3) предельному соотношению

$$
\lim _{j \rightarrow \infty} \int_{\mathbb{B}^{n}}\left\|g_{j}(z)-m_{j}\right\|_{1} d z=0 .
$$

Из (5.4) мы заключаем, что

$$
\lim _{j \rightarrow \infty}\left[g_{j}(z)-m_{j}\right]=0
$$

по мере в единичном шаре $\mathbb{B}^{n}$ и, следовательно, без потери общности мы можем предполагать, что

$$
\lim _{j \rightarrow \infty}\left[g_{j}(z)-m_{j}\right]=0
$$

почти всюду в $\mathbb{B}^{n}$. Поэтому все элементы матриц $m_{j}$ ограничены,

$$
\left|m_{j}^{i, k}\right| \leqslant Q^{2 / n}+d_{j}, \quad i, k=1,2, \ldots, n,
$$

где $d_{j} \rightarrow 0$ при $j \rightarrow \infty$. Таким образом, без потери обшности мы можем также предполагать, что существуют пределы

$$
\lim _{j \rightarrow \infty} g_{j}(z)=\lim _{j \rightarrow \infty} m_{j}=G_{0} \in S_{q}(n), \quad q=Q^{2 / n},
$$

почти всюду в единичном шаре $\mathbb{B}^{n}$.

Остаток доказательства является таким же, как в теоремах 3.1 и 4.1. 


\section{§ 6. $V M O$ и асимптотическая линейность}

Пусть $U$ - область в $\mathbb{R}^{n}$, содержащая начало 0 , и пусть $v, w: U \rightarrow \mathbb{R}^{m}$ - отображения, не обязательно непрерывные. Мы пишем $v(x)=o(w(x))$, если для каждого $\varepsilon>0$ найдется окрестность $V$ начала такая, что $\|v(x)\| \leqslant \varepsilon\|w(x)\|$ для всех $x \in V \backslash\{0\}$. Здесь норма $\|\cdot\|$ не обязательно должна быть обычной евклидовой нормой. Функции $v$ и $w$ называются әквивалентны.ми при $x \rightarrow 0$, пишем

$$
v(x) \sim w(x)
$$

если

$$
\|v(x)-w(x)\|=o(\|w(x)\|+\|v(x)\|) .
$$

Легко показать, что отношение $v(x) \sim w(x)$ является отношением эквивалентности и равносильно каждому из соотношений

$$
\begin{aligned}
& v(x)-w(x)=o(v(x)), \\
& v(x)-w(x)=o(w(x)) .
\end{aligned}
$$

Кроме того, если $m=1$, то мы имеем обычную эквивалентность вешественных величин.

В дальнейшем обычная евклидова норма и обычное скалярное произведение в $\mathbb{R}^{m}$ обозначается через $|\cdot|$ и $(\cdot, \cdot)$ соответственно. В [28] было показано,что отношение эквивалентности $v(x) \sim w(x)$ относительно обычной евклидовой нормы равносильно следующим двум геометрическим условиям:

$$
\begin{aligned}
|v(x)| & \sim|w(x)|, \\
(v(x), w(x)) & \sim|v(x)||w(x)| .
\end{aligned}
$$

Первое означает эквивалентность длин векторов $v(x)$ и $w(x)$, а второе означает, что угол между векторами $v(x)$ и $w(x)$ стремится к нулю при $x \rightarrow 0$.

Мы пишем $v(x) \approx w(x)$ и говорим, что $v$ и $w$ имеют один и тот же порядок в начале, если

$$
c^{-1}|v(x)| \leqslant|w(x)| \leqslant c|v(x)|
$$

для некоторого $c \geqslant 1$ при $x \rightarrow 0$.

Оба отношения эквивалентности (6.1) и (6.7) являются квазиконформными инвариантами. Теперь мы готовы дать основные определения.

Отображение $f: U \rightarrow \mathbb{R}^{m}, f(0)=0$, называется асимптотически линейным в нуле, если для каждого $\alpha \in \mathbb{R} \backslash\{0\}$

$$
f(\alpha x) \sim \alpha f(x)
$$

при $x \rightarrow 0$ и

$$
f(x+z) \sim f(x)+f(z)
$$


при $x \rightarrow 0$ и $x+z \approx x \approx z$.

Теорема 6.1. Пусть $n \geqslant 3 u f: D \rightarrow \mathbb{R}^{n}, f(0)=0 \in D$, - непостоянное квазирегулярное отображсение, и пусть либо дилатационный тензор $G_{f}(x)$, либо матричная дилатация $M_{f}(x)$ имеют исчезающую среднюю осцилляцию в нуле. Тогда отображсние $f$ асимптотически линейно в начале.

В частности, отсюда мы получаем следующие следствия (см. [6]).

СлЕДСТВИЕ 6.1. Пусть $n \geqslant 3 u f: D \rightarrow \mathbb{R}^{n}, f(0)=0 \in D$, - непостоянное $Q$-квазирегулярное отображсение, и пусть либо дилатационный тензор $G_{f}(x)$, либо матричная дилатация $M_{f}(x)$ являются непрерывными или хотя бы аппроксимативно непрерывными в нуле. Тогда отображение $f$ асимптотически линейно в начале.

ДокАЗАТЕЛЬСтво тЕОРЕмы 6.1. Пусть, например, $G_{f}(x)$ имеет исчезающую среднюю осцилляцию в нуле, т.е.

$$
\lim _{\varepsilon \rightarrow 0} \frac{1}{\Omega_{n} \varepsilon^{n}} \int_{|x|<\varepsilon}\left\|G_{f}(x)-M_{\varepsilon}\right\|_{1} d x=0, \quad M_{\varepsilon}=\frac{1}{\Omega_{n} \varepsilon^{n}} \int_{|x|<\varepsilon} G_{f}(x) d x .
$$

Рассмотрим семейство $Q$-квазирегулярных отображений

$$
h_{\varepsilon}(z)=f(\varepsilon z) / \rho(\varepsilon) \text {, }
$$

где $\rho(\varepsilon)=L(0, f, \varepsilon)$ и $L$ такое же, как в $(2.17)$.

В дальнейшем мы предполагаем, что семейство (6.11) задано для всех $0<\varepsilon<\varepsilon_{0}$ и $z \in B(0, r(\varepsilon)), r(\varepsilon)=r_{0} / \varepsilon \rightarrow \infty$ при $\varepsilon \rightarrow 0$, где $r_{0}=\operatorname{dist}(0, \partial D)$.

1) Итак, предположим, что (6.8) не имеет места для отображения $f$. Тогда найдутся $\rho \in \mathbb{R} \backslash\{0\}$ и последовательность $x_{j} \rightarrow 0, x_{j} \in \mathbb{R}^{n} \backslash\{0\}$, такие, что

$$
\frac{\left|f\left(\rho x_{j}\right)-\rho f\left(x_{j}\right)\right|}{\left|f\left(\rho x_{j}\right)\right|+\left|\rho f\left(x_{j}\right)\right|} \geqslant \Delta>0 \text {. }
$$

Кроме того, мы можем дополнительно предполагать, что $\eta_{j}=x_{j} /\left|x_{j}\right| \rightarrow \eta_{0} \in \mathbb{R}^{n}$, $\left|\eta_{0}\right|=1$.

Положим $H_{j}=h_{\delta_{j}}$, где $\delta_{j}=\left|x_{j}\right| \rightarrow 0$ при $j \rightarrow \infty$. Как легко видеть, отображения $H_{j}$ имеют дилатационные тензоры $g_{j}(z)=G_{f}\left(\delta_{j} z\right)$. Последовательно выбирая $\varepsilon=p \delta_{j}, p, j=1,2, \ldots$, в $(6.11)$, мы получаем

$$
\lim _{j \rightarrow \infty} \frac{1}{B(0, p)} \int_{B(0, p)}\left\|g_{j}(z)-m_{j, p}\right\|_{1} d z=0, \quad m_{j, p}=\frac{1}{B(0, p)} \int_{B(0, p)} g_{j}(z) d z
$$

для каждого фиксированного $p=1,2, \ldots$

Отметим, что все элементы матрищ $g_{j}(z)$ и, следовательно, матрищ $m_{j, p}$ ограничены числом $Q^{2 / n}$. Поэтому, используя диагональный процесс, мы можем предполагать, что

$$
\lim _{j \rightarrow \infty} g_{j}(z)=G_{0}
$$


почти всюду в $\mathbb{R}^{n}$, где $G_{0} \in S_{q}(n), q=Q^{2 / n}$.

Далее, по принципу максимума для открытых отображений и по лемме 2.1 мы имеем

$$
\limsup _{j \rightarrow \infty} \max _{|x| \leqslant R}\left|H_{j}(z)\right| \leqslant C^{2} R^{\beta}
$$

по крайней мере для всех $R>\gamma=C^{2 Q}>1$, где $\beta=Q^{\frac{1}{n-1}}$. Таким образом, мы можем предполагать, что $H_{j}(z) \rightarrow h(z)$ локально равномерно (см. [24, с. 10]), где $h$ - некоторое $Q$-квазирегулярное отображение с $h(0)=0$ по теореме Решетняка.

Ясно, что $h$ не является константой, поскольку снова ввиду правого неравенства из $(2.20)$ мы имеем $|h(z)| \geqslant C^{-2}|z|^{\beta}$ для $0<|z|<C^{-2 Q}<1$, где $\beta=Q^{\frac{1}{n-1}}$.

Поэтому по (6.14) и по теореме $2.1 \mathrm{~h}$ имеет постоянный дилатационный тензор $G_{0}$ почти всюду в $\mathbb{R}^{n}$. Рассуждая так же, как при доказательстве леммы 3.1 , мы получаем по теореме Лиувилля, что $h$ является аффинным отображением, и так как $h(0)=0, h$ является линейньм отображением всего пространства $\mathbb{R}^{n}$.

В частности, линейность $h$ влечет

$$
\begin{aligned}
\lim _{j \rightarrow \infty} \frac{\left|f\left(\rho x_{j}\right)-\rho f\left(x_{j}\right)\right|}{\left|f\left(\rho x_{j}\right)\right|+\left|\rho f\left(x_{j}\right)\right|} & =\lim _{j \rightarrow \infty} \frac{\left|H_{j}\left(\rho \eta_{j}\right)-\rho H_{j}\left(\eta_{j}\right)\right|}{\left|H_{j}\left(\rho \eta_{j}\right)\right|+\left|\rho H_{j}\left(\eta_{j}\right)\right|} \\
& =\frac{\left|h\left(\rho \eta_{0}\right)-\rho h\left(\eta_{0}\right)\right|}{\left|h\left(\rho \eta_{0}\right)\right|+\left|\rho h\left(\eta_{0}\right)\right|}=0,
\end{aligned}
$$

поскольку $\left|h\left(\eta_{0}\right)\right| \geqslant C^{-1}>0$, и локально равномерная сходимость влечет так называемую непрерывную сходимость (см., например, [29, с. 268]).

Однако (6.16) противоречит (6.12).

2) Аналогично, предположим, что (6.9) не имеет места для отображения $f$. Тогда сушествуют последовательности $x_{j}, z_{j} \in \mathbb{R}^{n} \backslash\{0\}, x_{j}, z_{j} \rightarrow 0$ при $j \rightarrow \infty$, такие, что $x_{j}+z_{j} \approx x_{j} \approx z_{j}$ и

$$
\alpha_{j}=\frac{\left|f\left(x_{j}+z_{j}\right)-f\left(x_{j}\right)-f\left(z_{j}\right)\right|}{\left|f\left(x_{j}+z_{j}\right)\right|+\left|f\left(x_{j}\right)+f\left(z_{j}\right)\right|} \geqslant \Delta>0 .
$$

Кроме того, мы можем предполагать дополнительно, что

$$
y_{j}=\left(x_{j}+z_{j}\right) /\left|x_{j}+z_{j}\right| \rightarrow y_{0}, \quad \zeta_{j}=z_{j} /\left|x_{j}+z_{j}\right| \rightarrow \zeta_{0}, \quad \eta_{j}=x_{j} /\left|x_{j}+z_{j}\right| \rightarrow \eta_{0}
$$

при $j \rightarrow \infty$, где $y_{0}, \eta_{0}, \zeta_{0} \in \mathbb{R}^{n}, y_{0}=\eta_{0}+\zeta_{0}$ и $\left|y_{0}\right|=1$. Тогда, полагая $\delta_{j}=\left|x_{j}+z_{j}\right|$ и рассуждая, как в предыдущем пункте, мы получаем

$$
\begin{aligned}
\lim _{j \rightarrow \infty} \alpha_{j} & =\lim _{j \rightarrow \infty} \frac{\left|H_{j}\left(y_{j}\right)-H_{j}\left(\eta_{j}\right)-H_{j}\left(\zeta_{j}\right)\right|}{\left|H_{j}\left(y_{j}\right)\right|+\left|H_{j}\left(\eta_{j}\right)+H_{j}\left(\zeta_{j}\right)\right|} \\
& =\frac{\left|h\left(y_{0}\right)-h\left(\eta_{0}\right)-h\left(\zeta_{0}\right)\right|}{\left|h\left(y_{0}\right)\right|+\left|h\left(\eta_{0}\right)+h\left(\zeta_{0}\right)\right|}=0,
\end{aligned}
$$

поскольку $\left|h\left(y_{0}\right)\right| \geqslant C^{-1}>0$. Однако (6.18) противоречит (6.17). 


\section{Список литературы}

1. Гольдитейн B. М. О поведении отображений с ограниченным искажением при коэффициенте искажения, близком к единице // Сиб. матем. журн. 1971. Т. 12. С. 1250-1258.

2. Martio O., Rickman S., Väisälä J. Topological and metric properties of quasiregular mappings // Ann. Acad. Sci. Fenn. Ser. AI. 1971. V. 488. P. 1-31.

3. Решетняк Ю. Г. Пространственные отображения с ограниченным искажением. Новосибирск: Наука, 1982.

4. Ferrand J. Regularity of conformal mappings of Riemannian manifolds // Lect. Notes in Math. 1979. V. 743. P. 191-203.

5. Bojarski B., Iwaniec T., Kopiecki R. Riemannian Manifolds with Non-Smooth Metric Tensors and $Q C$-Maps // Proc. Sem. on Monge-Ampere Equations Related Topics, Firenze 1980. Roma, 1982. P. 123-165.

6. Gutlyanskii V. Ya., Martio O., Ryazanov V.I., Vuorinen M. On local injectivity and asymptotic linearity of quasiregular mappings. Preprint 122. Helsinki: Department of Mathematics, University of Helsinki, 1996.

7. John F., Nirenberg L. On functions of bounded mean oscillation // Comm. Pure Appl. Math. 1961. V. 14. P. 415-426.

8. Astala K., Gehring F. W. Injectivity the BMO norm and the universal Teichmüller space // J. Analyse Math. 1986. V. 46. P. 16-57.

9. Reimann H. M., Rychener Th. Funktionen beschränkter mittlerer Oscillation // Lect. Notes in Math. 1975. V. 487.

10. Garnett J. B. Bounded analytic functions. New York etc.: Academie Press, 1981.

11. Saks S. Theory of the integral. New York: Dover Publ. Inc., 1964.

12. Bellmann R. Introduction to Matrix Analysis. New York etc.: McGraw-Hill Book Comp., 1970.

13. Ahlfors L. On a class of quasiconformal mappings // Österreich. Acad. Wiss. Math. Naturw. K1. S. BII. 1976. V. 185. P. 5-10.

14. Ahlfors L. Quasiconformal deformations and mappings in $\mathbb{R}^{n} / /$ J. Analyse Math. 1976. V. 30. P. 74-97.

15. Bers $L$. On a theorem of Mori and the definition of quasiconformality // Trans. Amer. Math. Soc. 1957. V. 84. P. 78-84.

16. Боярский Б.В. Обобщенные решения ситемы дифференциальных уравнений первого порядка эллиптического типа с разрывными коэффициентами // Матем. сб. 1957. T. 43(85). C. $451-503$.

17. Lehto O., Virtanen K. Quasikonforme Abbildungen. Berlin etc.: Springer-Verlag, 1965.

18. Iwaniec T., Kopiecki $R$. Stability in the differential equations for quasiregular mappings // Lect. Notes in Math. 1980. V. 798. P. 203-214.

19. Gutlyanskii V., Martio O., Ryazanov V., Vuorinen M. Convergence theorems for quasiregular mappings in the space. Preprint 105. Helsinki: Department of Mathematics, University of Helsinki, 1996.

20. Martio O, Rickman S., Väisälä J. Definitions for quasiregular mappings // Ann. Acad. Sci. Fenn. Ser. AI. 1969. V. 448. P. 1-40.

21. Решетняк Ю. Г. Локальная структура отображений с ограниченным искажением // Сиб. матем. журн. 1969. Т. 10. №6. С. 1319-1333.

22. Vuorinen M. Conformal Geometry and Quasiregular Mappings // Lect. Notes in Math. 1988. V. 1319.

23. Зорич В.А. Теорема Лаврентьева о квазиконформных отображениях пространства // Матем. сб. 1967. Т. 74(116). № 3. С. 417-433. 
24. Martio O., Rickman S., Väisälä J. Distortion and singularities of quasiregular mappings // Ann. Acad. Sci. Fenn. Ser. AI. 1970. V. 465. P. 1-13.

25. Bojarski B., Iwaniec T. Another approach to Liouville theorem // Math. Nachr. 1982. V. 107. P. 253-262.

26. Gehring F. W. Rings and quasiconformal mappings in space // Trans. Amer. Math. Soc. 1962. V. 103. P. 353-393.

27. Tukia P., Väisälä J. A remark on 1-quasiconformal maps // Ann. Acad. Sci. Fenn. Ser. AI. 1985. V. 10. P. 561-562.

28. Gutlyanskii V., Martio O., Ryazanov V., Vuorinen M. On asymptotical behavior of quasiconformal mappings in the space. Preprint 83. Helsinki: Department of Mathematics, University of Helsinki, 1995.

29. Dugundji J. Topology. Boston: Allyn and Bacon Inc., 1966.

Математический факультет

Поступило в редакцию

университета Хельсинки, Финляндия;

31.VII.1997

Институт прикладной математики

и механики НАН Украины, г.Донецк 